\title{
Radiation hardness tests of Avalanche Photodiodes for FAIR, NICA, and CERN SPS experiments
}

\author{
V. Mikhaylov*† \\ Nuclear Physics Institute, Czech Academy of Sciences, Řež, Czech Republic \\ Czech Technical University in Prague, Faculty of Nuclear Sciences and Physical Engineering, \\ Prague, Czech Republic \\ National Research Tomsk Polytechnic University, Department of Electronics and Automation of \\ Nuclear Plants, Tomsk, Russia (on leave from)
}

A. Kugler, S. Kushpil, V. Kushpil, O. Svoboda, P. Tlustý

Nuclear Physics Institute, Czech Academy of Sciences, Řež, Czech Republic

\section{Ladygin}

Joint Institute for Nuclear Research, Laboratory of High Energy Physics, Dubna, Russia

\begin{abstract}
Modern avalanche photodiodes with high gain are excellent device candidates for the light readout from detectors used for the high energy physics experiments. We report the results of the APDs radiation hardness study. Properties of APDs manufactured by Ketek and Hamamatsu companies has been studied in terms of internal defects accumulation. Test setups for offline and online APD irradiation measurements are described. Simplified models estimating contribution of various noise components are discussed in comparison with data achieved by static and dynamic characteristics analysis. The results of the APDs investigations after irradiation using secondary neutrons from the cyclotron facility U120M at the Nuclear Physics Institute of CAS in Řež are presented.
\end{abstract}

The European Physical Society Conference on High Energy Physics

22-29 July 2015

Vienna, Austria

\footnotetext{
${ }^{*}$ Speaker.

†email: mikhaylov@ujf.cas.cz
} 


\section{Introduction}

The idea of the very forward calorimeter which provides high and uniform energy resolution was already successfully applied as the Projectile Spectator Detector at NA61 experiment in CERN [1]. The very similar calorimeters are being designed and built for the future high energy nuclear physics facilities, such as the Projectile Spectator Detector for the CBM experiment at the future FAIR facility [2], the Forward Wall Detector for the Baryonic Matter Spectrometer (BM@N) [3] and the Multi Purpose Detector at the future NICA facility [4]. Those calorimeters allow the detection of the non-interacting nucleons and fragments emitted at very low polar angles in forward direction in heavy-ion collisions. It will be used to determine the collision centrality and the orientation of an event plane by measuring of the spatial position and energy of these nucleons and fragments.

As an example, we can discuss the Projectile Spectator Detector being built for the CBM experiment. It is a full compensating modular lead-scintillator calorimeter comprising 44 individual modules, each consists of 60 lead/scintillator layers with a surface of $20 \times 20 \mathrm{~cm}^{2}$ [5]. The scintillation light is read out via wavelength shifting fibers by Avalanche PhotoDiodes (APD). The main advantages of APDs are: very compact sizes, low bias voltage, gain comparable to PMT, relatively low price, insensitivity to magnetic field and absence of nuclear counter effect (due to the pixel structure). Generally, APDs have the following properties: pixel density about $10^{4}-2 \times 10^{4} \mathrm{~mm}^{-2}$, size of $3 \times 3 \mathrm{~mm}^{2}$, high dynamical range from 5 to 15000 photo electrons, photon detection efficiency of about $15 \%$, high counting rate of about $10^{5} \mathrm{~Hz}$.
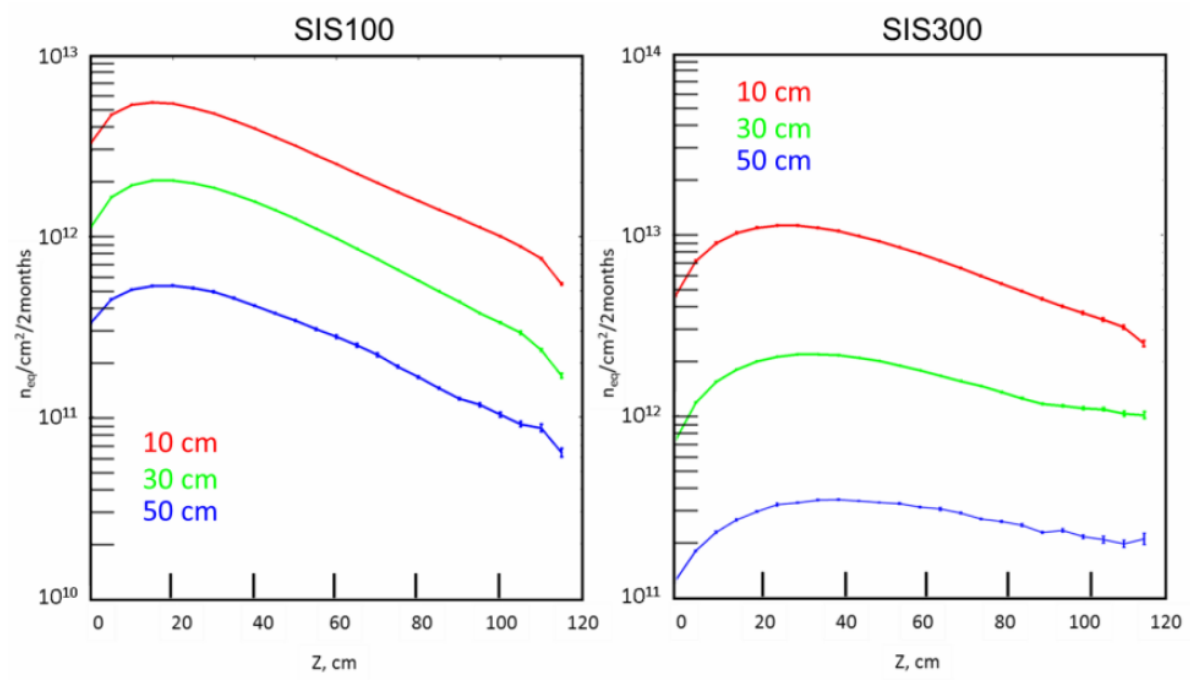

Figure 1: Distributions of the neutron flux through the PSD calorimeter at various radius [6].

The motivation of radiation resistance tests for hadron calorimeters at the current and future experiments is the very high intensity of the ion beams. The overall radiation dose deposited in the PSD and the neutron flux (Fig. 1) were simulated by FLUKA [6] for CBM and PSD geometry and material budget for SIS100 and SIS300 [5]. The simulation shows that radiation dose is not critical for scintillators itself. However, the most crucial effect could be the APD degradation caused by 
the neutron flux trough the rear side of PSD calorimeter. According to FLUKA simulation the flux near the beam hole might achieve $10^{12}$ neutrons $/ \mathrm{cm}^{2}$ for beam energy $4 \mathrm{AGeV}$ and about $4 \times 10^{12}$ neutrons $/ \mathrm{cm}^{2}$ for beam energy $35 \mathrm{AGeV}$ and two months of $\mathrm{CBM}$ run at the beam rate $10^{8}$ ions/sec. Therefore, the main requirement for APDs for PSD CBM is the radiation hardness to neutron fluxes up to $10^{13}$ neutrons $/ \mathrm{cm}^{2}$.

\section{Tests of irradiated APDs with LED and cosmics}

Modern investigation of single-photon detectors is not possible without single-photon sources. The common laboratory source of photons is Light Emitting Diode (LED). Main advantage of LED is the possibility to apply the method of synchronous detection of the APD test signal. It allows to investigate the APD after irradiation in the close to single-photon mode of operation, when signal to noise ratio is very low and can be calculated using big statistics of single photon events. The method allows to investigate threshold variation of APD photon detection. Cosmic rays are considered as the low cost replacement for conduction of an experiment with APDs involving accelerator facilities. It allows to employ high energetic muons (MIP particles) to test APDs in generally any experimental laboratory with the similar source of particles. The disadvantage of this method is low data rate, which is not important on stage of R\&D.

The APDs produced by Ketek and Hamamatsu manufacturers were irradiated at the Cyclotron facility of NPI Řež with $35 \mathrm{MeV}$ secondary neutron beam [7]. Neutron absorbed doses are $\mathrm{F}=2.5 \times 10^{12} \mathrm{n}_{\mathrm{eq}} / \mathrm{cm}^{2}$ for Ketek APD and $\mathrm{F}=6.5 \times 10^{10} \mathrm{n}_{\mathrm{eq}} / \mathrm{cm}^{2}$ for Hamamatsu APD. Doses were measured by the special online dosemeter device based on PIN diode calibrated for $1 \mathrm{MeV} \mathrm{n}$ eq $/ \mathrm{cm}^{2}$; the temperature during the irradiation and measurements was $22 \pm 0.5^{\circ} \mathrm{C}$ [8].
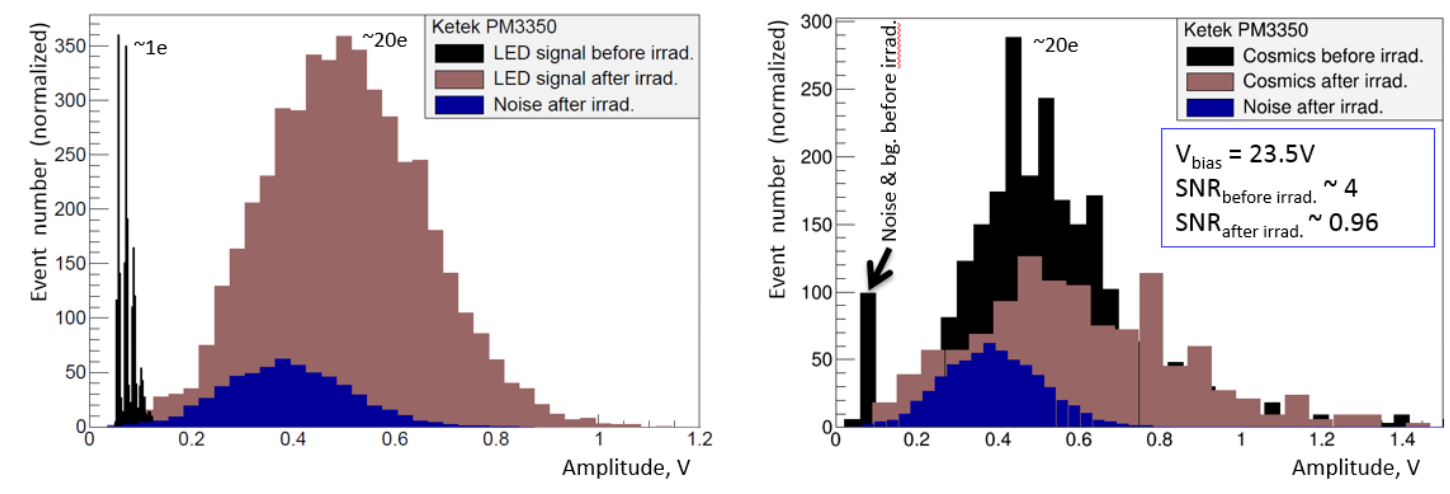

Figure 2: Test results of Ketek APD with LED (left) and cosmic muons (right).

Figure 2 shows the results of the studies of the APD PM3350 produced by Ketek [9] with LED (left) and with cosmic muons (right). The noise signal after the irradiation was measured when the LED or cosmics triggers were turned off. The noise before irradiation is visible as the very first peak pedestal on the signal before irradiation histogram. The multiple signal peaks around $0.05 \mathrm{~V}$ represent the clear individual single, double and higher orders photon peaks within the LED measurement before irradiation (Fig. 2, left). The Ketek APD is unable to resolve single photons 
after irradiation due to high noise level of $\sim 15$ photo electrons (p.e.). Figure 2 (right) shows that the averaged value of the detector signal amplitude is $\sim 0.4 \mathrm{~V}$ corresponding to $\sim 20$ p.e. The signal and noise peaks for irradiated Ketek APD are very close to each other which makes difficult the signal from noise separation.
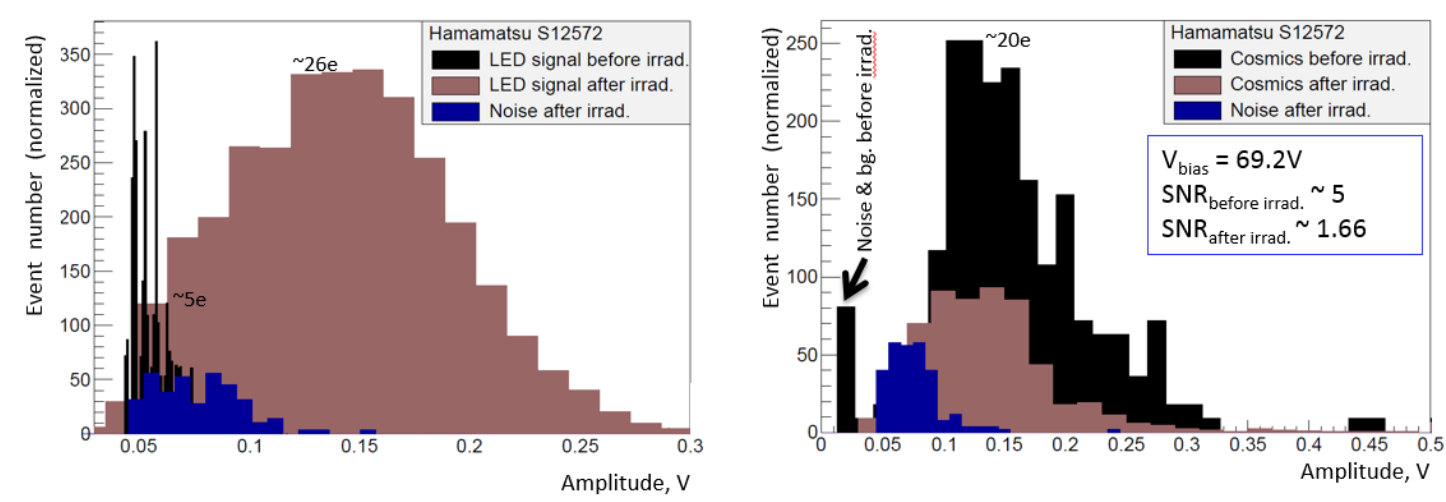

Figure 3: Test results of Hamamatsu APD with LED (left) and cosmic muons (right).

Figure 3 shows the results of the studies of the APD S12572-010P produced by Hamamatsu [10] with LED (left) and with cosmic muons (right). The marks in Figure 3 are the same as in Figure 2. We can see the good separation of the LED-induced single photon peaks for Hamamatsu APD before irradiation as in the case of Ketek APD. The averaged value of the LED signal amplitude from Hamamatsu APD after irradiation is $\sim 0.18 \mathrm{~V}$ corresponding to $\sim 26$ p.e. (Fig. 3, left). It is well separated from the noise peak $\sim 6$ p.e. The averaged value of the signal amplitude from Hamamatsu S12572-010P, obtained with cosmics, is $\sim 0.12 \mathrm{~V}$ corresponding to $\sim 20$ p.e. (Fig. 3, right). The signal from APD does not change dramatically and it is well separated from the noise after irradiation. However, we must note that the LED and cosmics test were done for Hamamatsu APD after the irradiation with neutron fluence of $30-50$ times less than for Ketek PM3350. It will be necessary to perform the tests for the Hamamatsu APD irradiated with the dose $\sim 10^{12}$ as well as more detailed studies with various doses.

\section{Advanced measurement techniques for APD investigation}

We carried out two additional APD investigation procedures, namely measurement and analysis of the Current Voltage Characteristics (I-V) and Capacitance Frequency Characteristics (CF). The reverse-biased I-V characteristic of P-N junction provides information concerning the generation-recombination process in the silicon bulk. This characteristic allows to estimate the influence of irradiation on noise performance of the detector. Results of C-F measurement are processed with help of a simplified model, where the ratio of the average lifetime of minority carriers to their average concentration is the main parameter characterizing the change in the junction after irradiation. This parameter provides a qualitative picture of the processes dynamics of the traps generation in silicon. The second technique based on the switching charge measurement gives an information about the lifetime of minority carriers in the base of PN junction. 

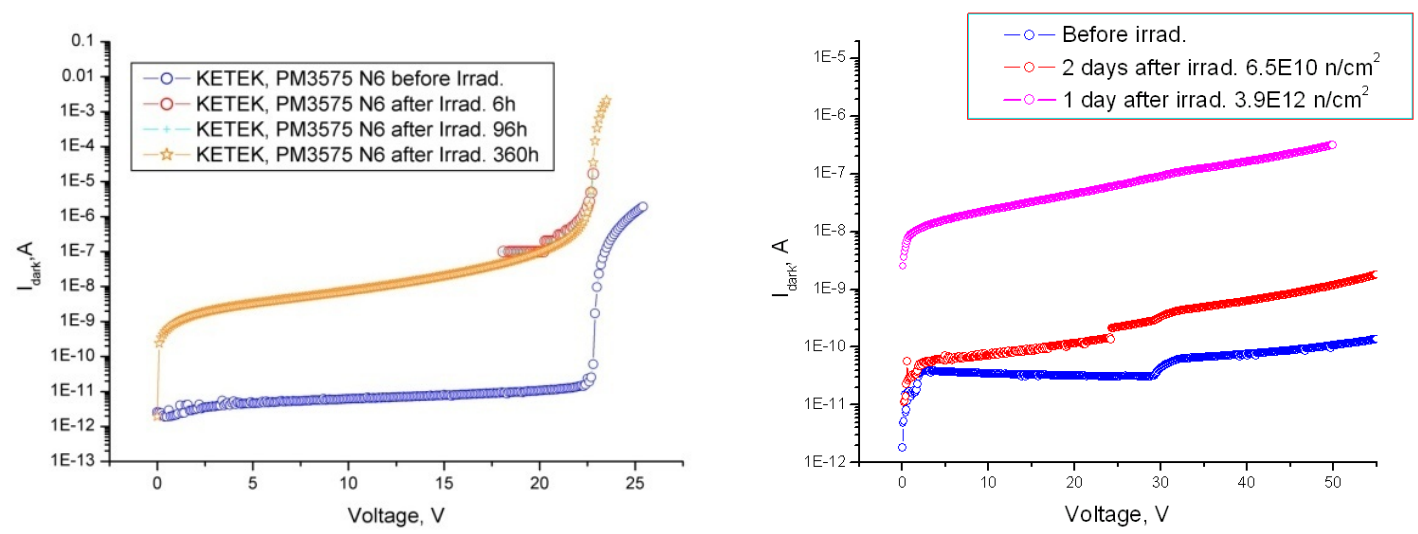

Figure 4: I-V curves of Ketek (left) and Hamamatsu (right) APDs before and after irradiation.

I-V characteristics of Ketek and Hamamatsu APDs are showing the increase of the dark current $\mathrm{I}_{\text {dark }}$ in $\sim 10^{3}$ times right after irradiation by dose of $\sim 10^{12} \mathrm{n}_{\mathrm{eq}} / \mathrm{cm}^{2}$ (Fig. 4). Due to self-annealing $\mathrm{I}_{\text {dark }}$ of Ketek APD decreased a bit in a week after the irradiation (Fig. 4, left). Hamamatsu APD was initially irradiated with a much smaller dose of $\sim 10^{10} \mathrm{n}_{\mathrm{eq}} / \mathrm{cm}^{2}$ leading to the increase of Idark in $\sim 10$ times near the operating voltage, which is still a significant change (Fig. 4, left).

New method of C-F data analysis is based on the dynamical process describing variation of nonequilibrium carriers due to recharge of traps' levels in silicon volume [11]. For the measured capacitance $\mathrm{C}$ variation of nonequilibrium carriersâĂ is described by equation (3.1). Afterwards, one can achieve the equation (3.2) by applying simple substitutions:

$$
\begin{aligned}
\frac{\partial \Delta n}{\partial t} & =\frac{1}{e} \cdot \frac{\partial J_{\mathrm{n}}}{\partial x}+\frac{\Delta n}{\tau} \\
\frac{1}{C}(f) & =\frac{1}{e} \cdot \frac{\langle\tau\rangle}{\left\langle N_{\mathrm{t}}\right\rangle} \cdot \Delta \varphi \cdot f
\end{aligned}
$$

where e is elementary charge, $J_{\mathrm{n}}$ is the current through p-n junction, $N_{\mathrm{t}}$ is traps levels concentration and $\Delta \varphi$ - voltage modulation applied to p-n junction at frequency $f$.

Achieved equation (3.2) describes the dependence of traps' levels in semiconductor volume on the capacitance of p-n junction and can be extremely useful for the C-F characteristics analysis [12].

The C-F characteristics of both Hamamatsu and Ketek APDs show the capacitance increase for high frequencies and the capacitance decrease for low frequencies after irradiation (Fig. 5). Considering the $1 / C \sim f$ model, this effect can be related respectively to the increase of shortliving traps amount and the decrease of long-living traps amount. The delimiting value of traps lifetime to be considered as short- or long-living is $2.5 \mu$ s for Ketek APD (Fig. 5, left). Process in Hamamatsu APD is different, probably due to its very complicated internal structure. Capacitance and therefore traps amount increased significantly for the whole frequency range right after the first irradiation by $\sim 10^{10} \mathrm{n}_{\mathrm{eq}} / \mathrm{cm}^{2}$, while no big impact was observed after increase of the dose by roughly two orders of magnitude (Fig. 5, right). 

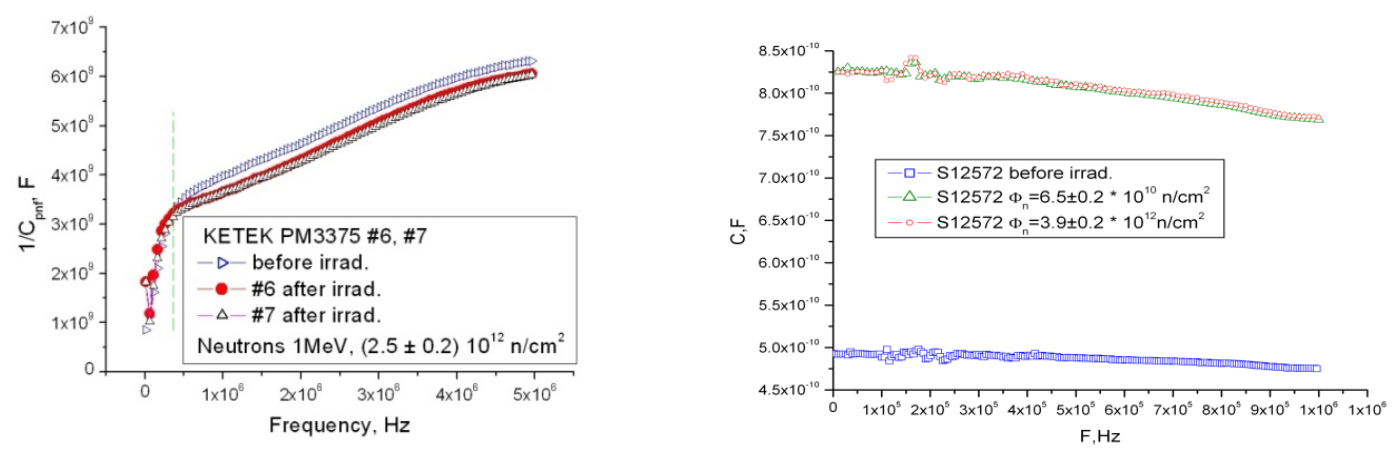

Figure 5: C-F curves of Ketek (left) and Hamamatsu (right) APDs before and after irradiation.

\section{Conclusion}

The studies of detectors properties for APDs produced by Ketek and Hamamatsu have been performed before and after irradiation by neutrons from the cyclotron facility U120M at NPI of ASCR in Řež. Internal noise of APDs dramatically increase after irradiation, that leads to inability to detect single photons. After irradiation signal and noise peaks are overlapping for Ketek PM3350 $\left(\mathrm{F}=2.5 \times 10^{12} \mathrm{n}_{\mathrm{eq}} / \mathrm{cm}^{2}\right)$. For significantly lower dose they are well separated in case of Hamamatsu S12572-010P $\left(\mathrm{F}=6.5 \times 10^{10} \mathrm{n}_{\mathrm{eq}} / \mathrm{cm}^{2}\right)$. Based on C-V and C-F analysis we assume that defects in Silicon volume produce the increase of high frequency noise. The results are certainly important to design the detectors with APD light readout for high energy physics experiments.

\section{Acknowledgments}

The authors thank the NPI cyclotron and neutron generators staff for excellent beam conditions and service. This work was supported by the European Community FP7 Capacities, contract HadronPhysics3 no.283286, LG12007 of the Ministry of Education of the Czech Republic and M100481202 of the Academy of Sciences of the Czech Republic grants, by project MSMT LG14004 of Cooperation Program between JINR and NPI in 2014-2015 and RFBR under grant No13-02-00101a, partially by TPU P.L.U.S. program.

\section{References}

[1] N. Abgrall et al. [NA61 Collaboration], JINST 9, P06005 (2014) [arXiv:1401.4699 [physics.ins-det]].

[2] The CBM experiment at FAIR facility [CBM Collaboration], http://www.fair-center.eu/public/experiment-program/nuclear-matter-physics/cbm.html.

[3] T. O. Ablyazimov et al. [BM@N Collaboration], Conceptual Design Report of BM@N, http://nica.jinr.ru/files/BM@N/BMN-CDR.pdf

[4] V. D. Kekelidze et al. [NICA Collaboration], Design and Construction of Nuclotron-based Ion Collider fAcility. Conceptual Design Report, http://nica.jinr.ru.

[5] F. Guber et al. [CBM Collaboration], Technical Design Report for the CBM Projectile Spectator Detector (PSD), https://repository.gsi.de/record/109059. 
[6] A. Senger, FLUKA Rechnungen fÃijr das CBM Experiment an FAIR, 77th Annual Meeting of the DPG and DPG Spring Meeting.

[7] M. Štefánik et al., Neutron Spectrum Determination of the p(35 MeV)-Be Source Reaction by the Dosimetry Foils Method, Nuclear Data Sheets 119, 422 (2014).

[8] V. Kushpil, S. Kushpil, Z. Huna, A simple device for the measurement of kerma based on commercial PIN photo diodes, EPJ Web of Conferences 24 (2012) 07008

[9] Ketek PM3350 Datasheet, http://www.ketek.net/products/sipm/pm3350/.

[10] Hamamatsu S12572-010P Datasheet, http://www.hamamatsu.com/jp/en/S12572-010P.html.

[11] E. Schibli and A. G. Milnes, Effects of deep impurities on $n+p$ junction reverse-biased small-signal capacitance, Solid-State Electronics 11, 323-334 (1968).

[12] V. Kushpil, V. Mikhaylov, A. Kugler, S. Kushpil, O. Svoboda and P. Tlustý, Radiation hardness investigation of avalanche photodiodes for the Projectile Spectator Detector readout at the Compressed Baryonic Matter experiment, NIMA 787, 117-120 (2015). 